\title{
Impacto psicológico da pandemia em estudan- tes universitários e a Linha de Apoio Psicológi- co SOS COVID-19 (APsi-UMinho e EPsi)
}

\author{
https://doi.org/10.21814/uminho.ed.24.2
}

\section{Eugénia Ribeiro}

Eugénia Ribeiro (ORCID: 0000-0002-8749-7130) é doutorada em Psicologia Clínica, Professora Auxiliar da Escola de Psicologia da Universidade do Minho e investigadora da Unidade de Psicoterapia e Psicopatologia - CIPsi, da mesma Escola. É atualmente Diretora de curso do Mestrado Integrado em Psicologia e coordenadora da Unidade de Intervenção da Associação de Psicologia (APsi-UMinho). Tem desenvolvido investigação focada nos processos relacionais em psicoterapia.

\section{Ana Rita Pereira}

Ana Rita Pereira tem o Mestrado Integrado em Psicologia pela Universidade de Coimbra. É psicóloga e colaboradora da Associação de Psicologia da Universidade do Minho (APsi-UMinho) da Escola de Psicologia.

\section{Miguel M. Gonçalves}

Miguel Gonçalves (ORCID: 0000-0003-2575-7221) é Professor Catedrático do Departamento de Psicologia Aplicada da Escola de Psicologia da Universidade do Minho e investigador da Unidade de Psicoterapia e Psicopatologia - CIPsi, da mesma Escola. Atualmente é Presidente da Escola de Psicologia, tendo sido diretor do Centro de Investigação em Psicologia e Vice-Presidente da Escola entre 2013 e 2017. Entre 2010 e 2015 foi Editor Associado da Revista Psychotherapy Research (Routledge). 


\section{Adriana Sampaio}

Adriana Sampaio (ORCID: 0000-0001-7347-1282) é licenciada e doutorada em Psicologia pela Universidade do Minho e tem o Mestrado Integrado em Medicina pelo Instituto de Ciências Biomédicas Abel Salazar. É Professora Auxiliar da Escola de Psicologia e diretora do Laboratório de Neurociência Psicológica - CIPsi, da mesma Escola, da qual é atualmente Vice-Presidente. É Presidente da Associação de Psicologia (APsi-UMinho), da Escola de Psicologia da Universidade do Minho. 


\section{RESUMO}

Neste capítulo apresentamos a linha de apoio psicológico SOS COVID-19, da iniciativa da APsi-UMinho, criada com o propósito de responder às necessidades da comunidade UMinho, emergentes no contexto da pandemia COVID-19. Com efeito, a literatura empírica tem demonstrado, a propósito do impacto psicológico de epidemias passadas e da pandemia COVID-19, que a comunidade estudantil universitária representa um dos grupos de risco para o desenvolvimento de perturbação psicológica que merece particular atenção. Este conhecimento justifica o planeamento e implementação de ações atempadas e dirigidas para intervenções em situações de crise e para a prevenção do agravamento de problemas emergentes, que se descrevem neste capítulo.

\section{INTRODUÇÃO}

Em dezembro de 2019, uma nova estirpe de um coronavírus foi detetada em Wuhan, província de Hubei, China. Esse vírus foi nomeado pela OMS (World Health Organization, 2020) como SARS-CoV-2 e a doença, COVID-19 (COronaVIrus Disease-19). A COVID-19 transmitiu-se rapidamente por todo o mundo, levando a Organização Mundial de Saúde a decretar, em março de 2020, um estado de pandemia. Em Portugal foi sendo implementado progressivamente um conjunto de medidas de prevenção regional e nacional de saúde pública e a 18 de março, declarado o estado de emergência. Estas medidas incluíram o encerramento generalizado de estabelecimentos de ensino (escolas, creches, estabelecimentos de Ensino Superior), bem como de espaços públicos não essenciais e proibição de eventos públicos, juntamente com outras medidas como distanciamento físico, confinamento a casa, restrições de circulação nacional, entre outras medidas que visaram conter a propagação da infeção (European Centre for Disease Prevention and Control (ECDC), 2020).

Ainda antes de ser declarado o estado de emergência, a 7 de março, a Universidade do Minho, após confirmação de um caso de infeção por SARS-CoV-2 na instituição e por recomendação das Autoridades de Saúde, decidiu a suspensão de aulas presenciais e o encerramento dos seus serviços, que incluíam bibliotecas e residências universitárias, proibição de deslocações em serviço, obrigando muita da comunidade 
UMinho a permanecer/regressar às suas casas. Estas medidas que foram anunciadas num fim de semana obrigaram à rápida reorganização de vários serviços e equipamentos da instituição e implicaram a mobilização de toda a comunidade UMinho num contexto sem precedentes. A Universidade do Minho foi assim o primeiro estabelecimento de Ensino Superior a suspender as aulas presenciais em Portugal, numa altura em que os serviços de apoio psicológico ou outros, com foco na resposta às necessidades emergentes que se faziam sentir durante a pandemia (psicológicas, de apoio digital ou outras), estavam ainda em fase de desenvolvimento.

\section{IMPACTO PSICOLÓGICO DA PANDEMIA}

Em Portugal, como em quase todo o mundo, as medidas de contenção adotadas como forma de prevenir a propagação da doença ficaram marcadas socialmente pela quarentena ou confinamento social obrigatório, implicando a mudança nas rotinas de vida diária (e.g. teletrabalho, aulas online), a separação das pessoas das suas famílias e da comunidade em geral.

Como consequência destas medidas e, com base no conhecimento sobre o impacto das situaçóes epidémicas anteriores, antecipa-se que a experiência de isolamento, a incerteza e as preocupações em relação ao que o futuro reserva terão um impacto psicológico significativo, a curto e longo prazo, na saúde mental das populações que vivenciam a pandemia (e.g., Cao et al., 2020; Wang et al., 2020). Alguns fatores, como a duração da quarentena, associada ao medo da infeção ou medo da transmissão a outros, o acesso a informações inadequadas (fake news), a diminuição dos contactos sociais, a perda financeira e o estigma em relação aos contaminados ou familiares, têm sido referidos como preditores de problemas de saúde mental, estando associados a um aumento significativo do sofrimento psicológico (Brooks et al., 2020). Este impacto psicológico tem sido caracterizado na literatura por um aumento de sintomas de stress pós-traumático, ansiedade, irritabilidade, distúrbios de sono e sintomas depressivos (Brooks et al., 2020; Hawryluck et al., 2004; Li et al., 2020; Osório et al., 2017; Palinkas, 2012; Reynolds et al., 2008; Sandal et al., 2020). 


\section{INTERVENÇÃO PSICOLÓGICA EM CRISE EM CONTEXTO DE PAN- DEMIA}

O sofrimento psicológico e as exigências psicológicas derivadas da experiência inesperada da pandemia COVID-19 são compreensíveis à luz do racional teórico que enquadra a experiência de stress e de crise psicológica (Lazarus, 1991, 1999). A experiência de crise psicológica, definida como uma rutura nos padrões habituais de funcionamento do indivíduo, pode ser provocada por fatores internos (e.g. infeção) ou externos ao indivíduo (e.g. perda significativa, alteração de rotinas). No entanto, os acontecimentos vividos e potencialmente precipitantes não têm necessariamente uma relação linear com a experiência de crise psicológica. Assim, para além do contexto, o modo como a pessoa avalia os seus recursos (internos e externos) para lidar com os mesmos tem um papel fundamental na resposta emocional. Quanto maior o desequilíbrio percebido entre as exigências dos acontecimentos e os recursos, maior é a probabilidade de uma resposta de crise psicológica (Lazarus, 1999).

Em certa medida, face a situaçóes desconhecidas e inesperadas (quer de valência positiva, quer negativa), este desequilíbrio é esperado, ou seja, é normal que, temporariamente, as pessoas experienciem alguma vulnerabilidade e precisem de ajustar os seus padróes de resposta de modo a retomar o seu sentido de coerência e equilíbrio pessoal. Neste sentido, reações emocionais, cognitivas e comportamentais menos ajustadas são esperadas desde que apresentem um curso progressivo e transitório. Neste caso, as experiências de crise, ainda que breves e moderadamente desconfortáveis, podem ser percebidas como desafios e ser mobilizadoras de mudanças positivas e de renovação de recursos pessoais. A maioria das pessoas, mesmo face a situaçóes adversas, tende a ser capaz de mobilizar os seus recursos e, a seu tempo e ritmo, seguir percursos de adaptação e resiliência psicológica (Bonanno, 2005). No entanto, algumas pessoas, dependendo de fatores de risco e de vulnerabilidade, apresentam maior dificuldade em confrontar adequadamente situações que ameaçam a sua estabilidade emocional e cognitiva, podendo viver uma experiência de crise mais duradoura ou persistente (e.g., Ingram \& Luxton, 2005; Lazarus, 1999). Entre estas pessoas estão, por exemplo, as que apresentam história de sintomatologia ou perturbação psicológica e as que apresentam maior sensibilidade à problemática que a situação de vida inesperada reativa (e.g., perda, perigo), ou as 
que precisariam de ajuda para superar dificuldades emergentes em momentos críticos e que, por falta desse apoio, persistiram em respostas desajustadas.

O carácter inesperado e invulgar da pandemia COVID-19, a natureza altamente contagiosa da infeção, a falta de conhecimento sobre o vírus e seus processos de transmissão e a incerteza em torno dos procedimentos de tratamento e da possibilidade de uma vacina eficaz tornam este contexto potencialmente gerador de crise psicológica. Neste cenário invulgar, de múltiplas perdas reais ou antecipadas, de alteração das rotinas e confinamento dos espaços físicos e relacionais, em que a perceção de segurança pessoal, a diferentes níveis, pode estar ameaçada, é natural que muitas pessoas avaliem esta situação como ameaçadora da sua integridade física e psicológica e percebam os seus recursos e competências para lhe fazer face como insuficientes ou ineficazes. Deste modo, não é surpreendente que o impacto psicológico da experiência da pandemia e das medidas de proteção implementadas se manifeste sob a forma de sintomas de ansiedade, pela antecipação de diferentes níveis de perigo, e/ou de depressão, pela antecipação ou vivência de diferentes tipos de perdas. Embora possa ser difícil de aceitar, é importante compreender que em situações novas e inesperadas, ativadoras da perceção de perda de controlo sobre a própria vida e que desafiam o nosso equilíbrio pessoal, é normal sentir medo, insegurança, tristeza; é normal estar agitado, intolerante ou algo desorganizado e é normal não ter o mesmo rendimento intelectual, académico e profissional (Horowitz, 1986; Maunder et al., 2003). Estas respostas, sendo transitórias e moderadas, são normais por significarem necessidades temporariamente não satisfeitas (e.g. necessidade de proximidade de outros significativos, necessidade de segurança pessoal) e podem ter uma função de proteção, seja do sistema individual, familiar ou comunitário (e.g. o medo pode proteger de comportamentos demasiado arriscados e ativa mecanismos de proteção e defesa; a tristeza ativa mecanismos de contenção da energia). No entanto, estas respostas cognitivo-emocionais e comportamentais, se desreguladas e persistentes, podem sinalizar a emergência de sintomatologia psicológica mais grave ou o risco do agravamento de sintomatologia psicológica em pessoas já mais frágeis deste ponto de vista. Assim, em contexto de pandemia, as pessoas com maior dificuldade em lidar com mudanças repentinas no seu quotidiano, ou com história psiquiátrica prévia, têm maior risco de experienciar elevados níveis de ansiedade e/ou 
depressão do que as que entendem estas mudanças como desafios superáveis (Brooks et al., 2020; Dirkzwager et al., 2006; Huang et al., 2020). Estudos sobre epidemias anteriores mostram que alguns fatores parecem promover a resiliência das populações e o bem-estar psicológico nestas circunstâncias. Por exemplo, a ativação das redes de suporte, o apoio social fornecido pelas pessoas com as quais um indivíduo está confinado, bem como o contacto com pessoas significativas por meio de tecnologias da informação e comunicação (e.g. telefone e redes sociais) são fatores que estão inversamente correlacionados com a severidade da sintomatologia psicopatológica (Palinkas et al., 2004). Além disso, o evitamento da exposição excessiva a notícias sobre a COVID-19, a manutenção de um estilo de vida saudável, isto é, com rotinas de sono, alimentação, exercício físico e momentos de autocuidado, têm sido apresentados como medidas que ajudam a prevenir um maior impacto psicológico negativo.

\section{IMPACTO PSICOLÓGICO DA PANDEMIA EM ESTUDANTES UNIVER- SITÁRIOS}

Embora a literatura empírica sobre o impacto psicológico das epidemias e pandemias anteriores, identifique alguns grupos como sendo de maior risco, incluindo profissionais de saúde que estão na linha de frente do combate à infeção, pessoas com fatores de risco que foram infetadas, pessoas com perdas significativas devido à infeção por COVID-19 e pessoas previamente diagnosticadas com psicopatologia (Gardner \& Moallef, 2015; Inchausti $e t$ al., 2020; Lee et al., 2018), sabe-se que os estudantes universitários têm sido também identificados como um grupo particularmente suscetível ao sofrimento psicológico consequente deste tipo de situações (e.g., Ho et al., 2020; Salman et al., 2020). Estudos que avaliaram o impacto psicológico das epidemias SARS e MERS relatam um impacto significativo na saúde mental dos estudantes (Al-Tammemi et al., 2020; Wong et al., 2007). Desde o início da pandemia COVID-19, os estudos realizados com amostras de estudantes universitários, em diferentes países, têm encontrado evidência de grande instabilidade emocional, sintomas de ansiedade e de depressão e diversos problemas de adaptação no confronto com circunstâncias provocadas pela pandemia (e.g., Cao et al., 2020; S. Liu et al., 2020; Salman et al., 2020; Wang et al., 2020). A emergência e/ou o agravamento de sintomas psicológicos em estudantes universitários 
estão relacionados com diferentes fatores de risco, associados quer com acontecimentos e circunstâncias familiares, quer com as mudanças ao nível da vida académica. No primeiro caso, incluem-se as preocupaçóes com familiares infetados ou com maior risco de o serem (pais, avós), as preocupações com a perda ou instabilidade financeira pessoal e/ou familiar em consequência das medidas de confinamento e a falta de espaço pessoal em casa (Al-Tammemi et al., 2020; Irawan et al., 2020; Salman et al., 2020). As preocupações com a estabilidade financeira familiar associam-se com as preocupações com a vida académica, nomeadamente no que se refere ao pagamento de propinas ou ao pagamento de internet para acesso a aulas online (Gonzalez et al., 2020). Nesta vertente, alguns estudos têm mostrado que estudantes universitários provenientes de famílias com menor poder económico apresentam mais queixas com os custos da internet para suportar a aprendizagem, preocupaçóes com o pagamento de propinas, preocupaçóes com os resultados académicos e níveis de ansiedade superiores aos apresentados por estudantes que pertencem a famílias menos vulneráveis financeiramente (Gonzalez et al., 2020; Irawan et al., 2020). Congruentemente com estes estudos, Al-Tammemi, Akour, \& Alfalah (2020) verificaram que estudantes com mais dificuldades no acesso à aprendizagem online e com menor motivação para o ensino a distância apresentavam sofrimento psicológico mais severo. Outros fatores relacionados com a organização das atividades académicas têm sido relatados como fatores de risco que influenciam significativamente a saúde mental dos estudantes universitários. Entre estes fatores incluem-se: a alteração no formato de ensino de presencial para online, o afastamento das rotinas normais dos estudantes, quer relacionadas com o estudo e atividades letivas, quer relacionadas com as dinâmicas interpessoais associadas ao distanciamento dos colegas, amigos e professores (e.g. Irawan et al., 2020; Salman et al., 2020). A falta de suporte social é um dos fatores que se correlaciona fortemente com a experiência de ansiedade dos estudantes (Thompson et al., 2016), o que em época de pandemia se agrava pelas medidas de confinamento e proteção impostas não só aos estabelecimentos de ensino superior mas também à sociedade em geral.

Por outro lado, questóes relacionadas com a perceção de insegurança e de imprevisibilidade relacionada com o progresso académico têm sido também referidas na literatura como preditores de sintomatologia psicológica. A este respeito, por exemplo, 
um estudo longitudinal realizado com estudantes universitários chineses mostrou um aumento significativo da sintomatologia depressiva e ansiosa duas semanas após o período de confinamento, cujos fatores preditores foram a perceção de medidas sanitárias inadequadas, o nível mais elevado de graduação (justificado pelo medo de impacto negativo na finalização dos cursos) e a sintomatologia prévia (Li et al., 2020). Assim, a natureza inesperada e imprevisível das vivências académicas derivadas das medidas de proteção, da alteração de rotinas letivas e sociais e da organização dos processos de ensino-aprendizagem, a par do aumento da pressão para dar respostas imediatas às novas exigências e da duração imprevisível da pandemia e dos seus efeitos (Liu et al., 1997) tem sido relacionada com o agravamento da experiência de ansiedade e de burnout dos estudantes (Garfin et al., 2020).

\section{ESTRATÉGIAS USADAS PELOS ESTUDANTES PARA LIDAREM COM O SOFRIMENTO PSICOLÓGICO}

Estudos realizados com estudantes universitários durante a pandemia de COVID-19 mostraram que os estudantes usam diferentes estratégias para lidarem com situações geradoras de ansiedade. $\mathrm{O}$ recurso à espiritualidade e à religião, a aceitação da experiência, estratégias orientadas para lidar diretamente com as situaçóes ansiogénicas, uso de substâncias, estratégias distrativas e de planeamento de ações (Salman et al., 2020), assim como passar mais tempo em redes sociais para compensar o isolamento e o uso de medicação são algumas das estratégias relatadas pelos estudantes (Al-Tammemi et al., 2020). De acordo com Eisenberg e coautores (2012), as estratégias de confronto ativo (e.g., orientadas para lidar com as situaçóes perturbadoras, reenquadramento positivo, planeamento de ações, aceitação da experiência, procura de suporte emocional e procura de suporte informativo) são estratégias que se associam com melhores respostas face à adversidade, quando comparadas com as estratégias de evitamento (e.g., negação da experiência, uso de substâncias, desinvestimento comportamental, distração ou culpabilização) (Eisenberg et al., 2012). O agravamento das dificuldades e dos sintomas psicológicos dos estudantes tem também sido relacionado com o uso de estratégias de regulação mal adaptadas e disfuncionais, tais como o isolamento, o consumo de substâncias e a procrastinação (Gao et al., 2004). 
Uma resposta atempada e responsiva às necessidades manifestadas pelos estudantes que experienciam sofrimento psicológico é importante para corrigir estratégias de evitamento e diminuir o risco de agravamento de problemas de saúde mental mais graves. Alguns estudos têm mostrado que os estudantes universitários beneficiam de intervenção psicológica contingente à experiência epidémica como forma de gestão adequada das suas emoções e de ajustamento psicológico (Bai et al., 2005; Pan et al., 2005; Pang et al., 2003). São exemplo disso o impacto de intervenções em grupo com estudantes que vivenciaram o surto de SARS na China, traduzido numa diminuição da perceção de isolamento social em contexto de confinamento; na promoção do suporte social e na partilha de estratégias de confronto adequadas (Pan et al., 2005). Como já afirmamos, o suporte social real ou percebido durante situações de isolamento tem vindo a ser associado a menor sintomatologia de ansiedade e depressão (Bai et al., 2005; Cao et al., 2020; Kmietowicz, 2020; Tang et al., 2020; Xiao, 2020).

Em síntese, os vários estudos realizados em amostras de estudantes universitários sublinham a necessidade de prestar atenção ao impacto psicológico das adversidades geradas pela pandemia COVID-19 e recomendam que as instituições educativas promovam medidas sugeridas pela OMS para melhorar e promover a saúde mental dos seus estudantes (Al-Tammemi et al., 2020; Salman et al., 2020), prestem atenção à duração apropriada do confinamento e assegurem materiais e medidas adequados de controlo da infeção (Li et al., 2020). Num artigo publicado sob a forma de carta dos estudantes de medicina da Imperial College London, Ullah and Amin (2020) sublinham a intensificação das preocupações e ansiedade em tempos de incerteza provocados pela pandemia COVID-19, enfatizando o papel das universidades em mitigar este impacto psicológico negativo através de regular e atualizar as propostas de organização das atividades académicas e realçar a importância do suporte social e da comunicação interpessoal (Ullah \& Amin, 2020). Estas duas sugestões corroboram a importância da perceção de previsibilidade e de controlo sobre o que podem antecipar viver na academia, devolvendo assim aos estudantes um maior sentido de segurança. 


\section{A LINHA DE APOIO PSICOLÓGICO SOS COVID-19}

A necessidade de prestar apoio psicológico à comunidade académica no contexto da COVID-19, mantendo simultaneamente o respeito pelas medidas de contenção, levou à criação da primeira linha de apoio psicológico por telefone da Universidade Portuguesa, dirigida à comunidade académica. O objetivo dessa linha telefónica era ajudar a resolver problemas imediatos relacionados com o impacto psicológico e comportamental da COVID-19 e impedir o agravamento desse impacto a médio e longo prazo.

A fim de fornecer apoio psicológico imediato e gratuito aos estudantes e à comunidade académica, a 18 de março de 2020 a Associação de Psicologia da Universidade do Minho (APsi-UMinho) e a Escola de Psicologia lançaram a Linha de Apoio Psicológico SOS COVID-19. Este projeto propõe uma linha de intervenção psicológica em crise, com objetivos de prevenção do desenvolvimento de psicopatologia reativa aos desafios que a pandemia COVID-19 coloca à saúde mental da população. Este apoio tem a colaboração de especialistas e psicólogos afetos à APsi-UMinho e da Escola de Psicologia da Universidade do Minho, numa articulação estreita com psicólogos do Centro de Medicina Digital P5, da mesma Universidade e com as Câmaras Municipais de Braga e Guimarães. A linha funciona de segunda a sexta, das $9 \mathrm{~h}$ às $13 \mathrm{~h}$ e das $14 \mathrm{~h}$ às $18 \mathrm{~h}$.

Em situação de quarentena e de confinamento social, a linha telefónica implementada pela APsi-UMinho criou a oportunidade de acesso a um serviço psicológico breve e atempado, para os estudantes da Universidade do Minho, que em situação de fragilidade emocional, se veem impedidos de aceder a serviços de apoio psicológico presencial (e.g. em fase de quarentena e confinamento social), ou para quem o acesso à internet é limitado (e.g. estudantes que em tempo de pandemia vivem em ambientes com acesso mais difícil a redes de internet).

A linha de apoio psicológico SOS COVID-19 orienta-se por um modelo de intervenção em crise, diferenciado em função de problemas e necessidades psicológicas específicas, da autoria de investigadores e docentes da Escola de Psicologia da Universidade do Minho, em articulação com a APsi-UMinho. Este modelo de intervenção foi descrito e ilustrado num artigo publicado em junho de 2020, num número especial dedicado à investigação no âmbito da COVID-19 da revista internacional Counselling 
Psychology and Quartely (Ribeiro et al., 2020). A intervenção proposta neste modelo apoia-se nas diretrizes gerais da intervenção em crise psicológica (e.g. Meyer \& James, 2005), tendo como objetivo final contribuir para a estabilização cognitivo-emocional dos estudantes que recorrem a este apoio e promover a ativação dos seus recursos e competências adequadas para fazer face às situaçôes reais, ou percebidas como ameaçadoras do seu equilíbrio pessoal. A intervenção, embora breve, está estruturada em cinco passos: 1) apresentação pessoal e estabelecimento de uma ligação de confiança, 2) avaliação e identificação das preocupaçôes e problemas centrais; 3) identificação de recursos e possíveis soluçóes alternativas; 4) definição de um plano realista para implementar no imediato e 5) finalização da sessão de ajuda, assegurando que o/a estudante está estável, avaliando a necessidade de uma monitorização da necessidade de apoio psicológico e mantendo a disponibilidade da linha de apoio, caso o/a estudante sinta necessidade (Figura 1).

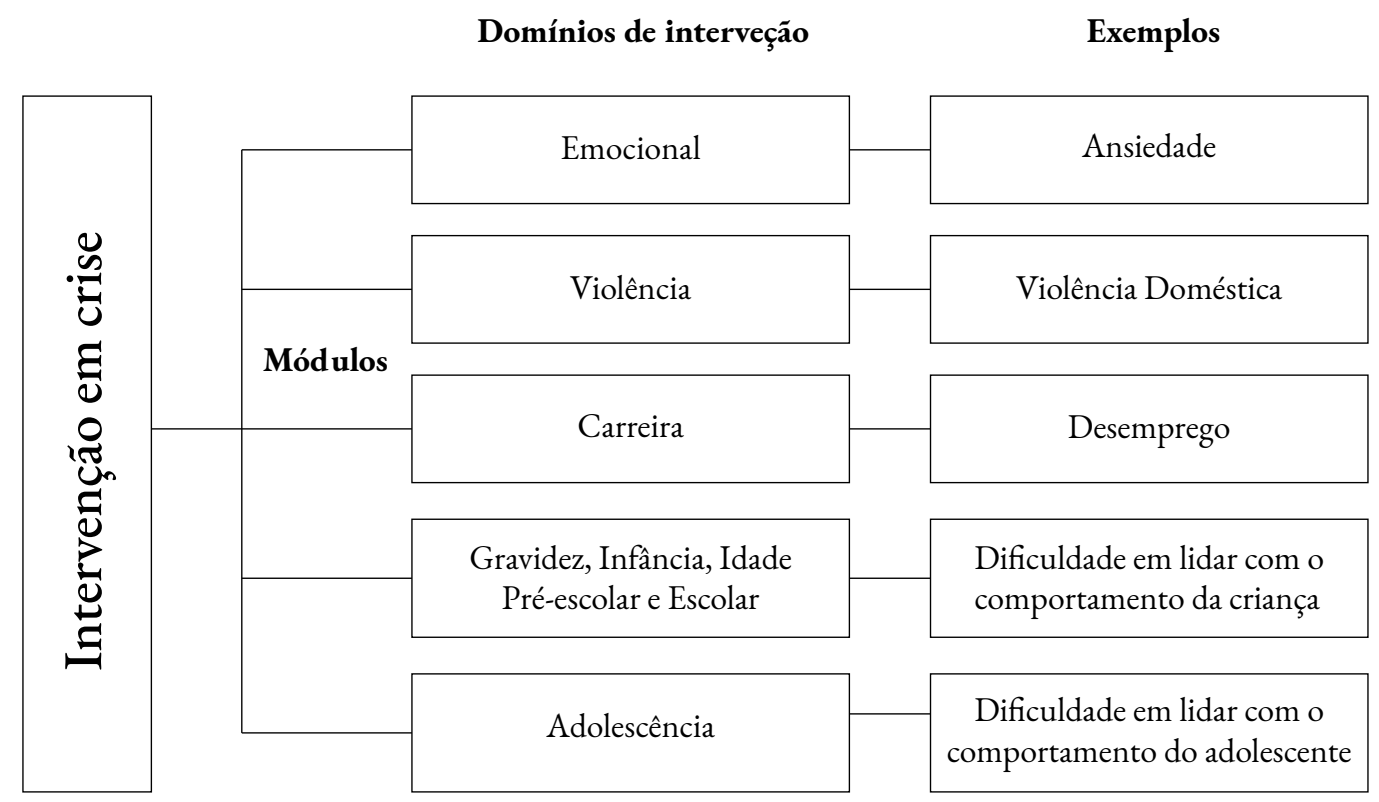

Figura 1. Modelo de Intervenção em Crise. Imagem adaptada de Ribeiro et al. (2020). (Ribeiro et al., 2020).

Desde março de 2020, 27 psicólogas credenciadas pela Ordem dos Psicólogos Portugueses (OPP), têm participado voluntariamente nesta linha de apoio, disponibilizando duas a três horas por semana para prestar apoio aos estudantes que recorram a esta linha. As psicólogas foram previamente integradas em equipas de intervenção 
especializadas em domínios específicos (e.g. psicoterapia com adultos, psicoterapia com crianças e adolescentes, intervenção de violência doméstica, intervenção educacional e de carreira). As psicólogas têm formação de base predominantemente cognitivo-comportamental, orientação teórica compatível com as diretrizes centrais do modelo de intervenção em crise implementado na linha de apoio.

Numa fase anterior à implementação da linha de apoio psicológico SOS COVID-19, estas psicólogas receberam treino em intervenção em crise psicológica, considerando as especificidades da pandemia COVID-19, apoiado por um manual de intervenção elaborado para este efeito pela equipa coordenadora da linha. Com o objetivo a supervisionar a intervenção implementada e apoiar as psicólogas que prestam serviço nesta linha, a equipa coordenadora dos vários módulos de intervenção especializada reúne semanalmente com todas as psicólogas para discussão de casos atendidos e das medidas implementadas.

\section{CARACTERIZAÇÃO DOS PEDIDOS E RESPOSTAS AOS ESTUDANTES NO CONTEXTO DA LINHA DE APOIO PSICOLÓGICO SOS-COVID-19}

Entre o dia 18-03-2020 e 23-06-2020 foram atendidas 80 chamadas, sendo 50\% das quais provenientes da comunidade académica da Universidade do Minho ( $47 \%$ de estudantes, $1,3 \%$ de docentes e 1,3\% de funcionários não docentes). As restantes $50 \%$ foram efetuadas por pessoas da comunidade envolvente da Universidade do Minho (Braga e Guimarães).

Os pedidos efetuados pelos estudantes da Universidade do Minho foram mais frequentes em março $(36,6 \%, n=15)$, abril $(31,7 \%, n=13)$ e maio $(24,4 \%, n=10)$, tendo decrescido substancialmente no mês de junho $(4,9 \%, n=2)$. A maioria destes pedidos foram efetuados por estudantes do sexo feminino (95\%).

Os motivos apresentados pelos estudantes no contexto deste atendimento em crise incluem, na sua maioria $(75,6 \%)$, problemas relacionados com a vida pessoal e familiar do/a estudante (e.g. dificuldades em lidar com as medidas de confinamento, cuidado de familiares, medos relacionados com a possibilidade de infeção, traduzidas na maioria dos casos em manifestações de ansiedade e angústia) e problemas relacionados com a 
vida académica (e.g. dificuldades com tarefas de estudo, avaliações, dúvidas em relação a procedimentos/futuro académico) (19,5\%). Uma menor percentagem dos pedidos referiu-se a pedidos de informaçôes sobre o atendimento da linha para os próprios ou familiares (5\%).

Entre as respostas de intervenção psicológica em crise, que se mostraram mais apropriadas aos problemas apresentados incluem-se: 1) escuta ativa/validação da experiência, securização e facilitação da regulação emocional (87,8\%); 2) informação/orientação académica ou outra (22\%); 3) monitorização via telefone da estabilidade emocional e organização comportamental, em geral com regularidade semanal (19,5\%); 4) encaminhamento para outros serviços para resolução de questôes académicas ou de saúde (e.g. psicoterapia online ou consulta médica, 19,5\%), seja via P5, seja via Serviço Nacional de Saúde. Alguns estudantes recorreram ao apoio das psicólogas da linha mais que uma vez $(19,5 \%)$.

\section{CONCLUSÃO}

O modelo de intervenção aqui apresentado foi desenvolvido com base na contribuição e expertise da Escola de Psicologia e APsi-UMinho e baseou-se nos modelos atuais de intervenção em crise, considerando as contribuições empíricas já existentes ao nível da caraterização do impacto psicológico na comunidade estudantil em contexto de pandemia COVID-19. Esta resposta emergiu num contexto em que ainda não existiam estruturas organizadas de apoio psicológico em crise em contexto de pandemia a nível nacional. Como refere a OMS (2020), a promoção da saúde mental deve ser considerada um componente central da resposta à COVID-19 como forma de amortecer os efeitos negativos da pandemia. De facto, a promoção da saúde mental promove a resiliência e permite manter a adesão às medidas de saúde pública e, simultaneamente, evitando sintomas e perturbaçóes psicopatológicos (IASC Reference Group on Mental Health and Psychosocial Support in Emergency, 2020). A APsi-UMinho através da linha de apoio psicológico SOS COVID-19 está empenhada em contribuir para ajudar os estudantes, assim como toda a comunidade académica, a lidarem com os desafios colocados pela pandemia COVID-19 à sua Saúde Mental, ajudando a identificar e suportar recursos adequados para lidarem com as dificuldades ou preocupaçóes 
imediatas, facilitar o autocuidado e informar/orientar para estruturas de apoio mais adequadas a problemas de natureza específica.

\section{REFERÊNCIAS BIBLIOGRÁFICAS}

Al-Tammemi, A. B., Akour, A., \& Alfalah, L. (2020). Is it Just About Physical Health? An Internet-Based CrossSectional Study Exploring the Psychological Impacts of COVID-19 Pandemic on University Students in Jordan Using Kessler Psychological Distress Scale. MedRxiv. https://doi.org/10.1101/2020.05.14.20102343.

Bai, Y.X., Gegan, T., Hai, H., Liu, Z. H., Wang, W. R., \& Wang,Z. G. (2005). Correlation between psychological changes of the community crowd and the social support in grave public health event. Inner Mongolia Med., $37(04), 295297$.

Bonanno, G. A. (2005). Resilience in the face of potential trauma. In Current Directions in Psychological Science. https://doi.org/10.1111/j.0963-7214.2005.00347.x.

Brooks, S. K., Webster, R. K., Smith, L. E., Woodland, L., Wessely, S., Greenberg, N., \& Rubin, G. J. (2020). The psychological impact of quarantine and how to reduce it: rapid review of the evidence. In The Lancet. https://doi.org/10.1016/S0140-6736(20)30460-8.

Cao, W., Fang, Z., Hou, G., Han, M., Xu, X., Dong, J., \& Zheng, J. (2020). The psychological impact of the COVID-19 epidemic on college students in China. Psychiatry Research. https://doi.org/10.1016/j. psychres.2020.112934.

Dirkzwager, A. J. E., Grievink, L., Van Der Velden, P. G., \& Yzermans, C. J. (2006). Risk factors for psychological and physical health problems after a man-made disaster: Prospective study. British Journal of Psychiatry. https://doi.org/10.1192/bjp.bp.105.017855.

Eisenberg, S. A., Shen, B. J., Schwarz, E. R., \& Mallon, S. (2012). Avoidant coping moderates the association between anxiety and patient-rated physical functioning in heart failure patients. Journal of Behavioral Medicine. https://doi.org/10.1007/s10865-011-9358-0.

European Centre for Disease Prevention and Control (ECDC). (2020). Joint European Roadmap towards lifting COVID-19 containment measures. https://ec.europa.eu/info/sites/info/files/communication - a european_roadmap_to_lifting_coronavirus_containment_measures_0.pdf.

Gao, Y., Xu, M. Z., \& Yang, Y. F. (2004). Research on coping style and related factors of college students during SARS outbreak. Chinese Medical Ethics, 02: 60-63., Chinese Me(02), 60-63.

Garfin, D. R., Silver, R. C., \& Holman, E. A. (2020). The Novel Coronavirus (COVID-2019) Outbreak: Amplification of Public Health Consequences by Media Exposure. In Health Psychology. https://doi. org/10.1037/hea0000875. 
Gonzalez, D., Karpman, M., Kenney, G. M., \& Zuckerman, S. (2020). Hispanic Adults in Families with Noncitizens Disproportionately Feel the Economic Fallout from COVID- 19. https:/www.urban.org/sites/ default/files/publication/102170/hispanic-adults-in-families-with-noncitizens-disproportionately-feel-theeconomic-fallout-from-COVID-19_0.pdf.

Hawryluck, L., Gold, W. L., Robinson, S., Pogorski, S., Galea, S., \& Styra, R. (2004). SARS control and psychological effects of quarantine, Toronto, Canada. Emerging Infectious Diseases. https://doi.org/10.3201/ eid1007.030703.

Ho, C. S., Chee, C. Y., \& Ho, R. C. (2020). Mental Health Strategies to Combat the Psychological Impact of COVID-19 Beyond Paranoia and Panic. Annals of the Academy of Medicine, Singapore.

Horowitz, M. J. (1986). Stress-response syndromes: A review of posttraumatic and adjustment disorders. Hospital and Community Psychiatry. https://doi.org/10.1176/ps.37.3.241.

Huang, L., Xu, F. M., \& Liu, H. R. (2020). Emotional responses and coping strategies of nurses and nursing college students during COVID-19 outbreak. In medRxiv. https://doi.org/10.1101/2020.03.05.20031898.

IASC Reference Group on Mental Health and Psychosocial Support in Emergency. (2020). Addressing Mental Health and Psychological Aspects of COVID-19 outbreak. Version 1.5.

Ingram, R. E., \& Luxton, D. D. (2005). Vulnerability-Stress Models. In B. L. Hankin \& J. R. Z. Abela (Eds.), Development of Psychopathology: A vulnerability stress perspective (pp. 32-46). Inc., Sage Publications.

Irawan, A. W., Dwisona, D., \& Lestari, M. (2020). No Title. KONSELI: Journal Bimbingan Dan Konseling (E-Journal), 7(1), 53-60.

Kmietowicz, Z. (2020). Rules on isolation rooms for suspected COVID-19 cases in GP surgeries to be relaxed. BMJ (Clinical Research Ed.). https://doi.org/10.1136/bmj.m707.

Lazarus, R. S. (1991). Emotion and adaptation. Oxford University Press.

Lazarus, R. S. (1999). Stress and emotion: A new synthesis. Springer.

Li, H. Y., Cao, H., Leung, D. Y. P., \& Mak, Y. W. (2020). The psychological impacts of a COVID-19 outbreak on college students in China: A longitudinal study. International Journal of Environmental Research and Public Health. https://doi.org/10.3390/ijerph17113933.

Liu, D., Diorio, J., Tannenbaum, B., Caldji, C., Francis, D., Freedman, A., Sharma, S., Pearson, D., Plotsky, P. M., \& Meaney, M. J. (1997). Maternal care, hippocampal glucocorticoid receptors, and hypothalamicpituitary-adrenal responses to stress. Science. https://doi.org/10.1126/science.277.5332.1659.

Liu, S., Yang, L., Zhang, C., Xiang, Y. T., Liu, Z., Hu, S., \& Zhang, B. (2020). Online mental health services in China during the COVID-19 outbreak. In The Lancet Psychiatry. https://doi.org/10.1016/ $\underline{\text { S2215-0366(20)30077-8. }}$. 
Maunder, R., Hunter, J., Vincent, L., Bennett, J., Peladeau, N., Leszcz, M., Sadavoy, J., Verhaeghe, L. M., Steinberg, R., \& Mazzulli, T. (2003). The immediate psychological and occupational impact of the 2003 SARS outbreak in a teaching hospital. CMAJ.

Meyer, R. ., \& James, R. K. (2005). Crisis Intervention Workbook. Thomson Brooks/Cole.

Osório, A. A. C., Rossi, N. F., Gonçalves, Ó. F., Sampaio, A., \& Giacheti, C. M. (2017). Psychopathology and behavior problems in children and adolescents with Williams syndrome: Distinctive relationships with cognition. Child Neuropsychology, 23(6). https://doi.org/10.1080/09297049.2016.1183607.

Palinkas, L. A. (2012). A Conceptual Framework for Understanding the Mental Health Impacts of Oil Spills: Lessons from the Exxon Valdez Oil Spill. Psychiatry: Interpersonal and Biological Processes, 75(3), 203-222.

Palinkas, L. A., Petterson, J. S., Russell, J. C., \& Downs, M. A. (2004). Ethnic differences in symptoms of posttraumatic stress after the Exxon Valdez oil spill. Prehospital and Disaster Medicine. https://doi.org/10.1017/ $\underline{S 1049023 X 00001552 .}$

Pan, P. J. D., Chang, S. H., \& Yu, Y. Y. (2005). A support group for home-quarantined college students exposed to SARS: Learning from practice. Journal for Specialists in Group Work. https://doi. org/10.1080/01933920500186951.

Pang, X., Zhu, Z., Xu, F., Guo, J., Gong, X., Liu, D., Liu, Z., Chin, D. P., \& Feikin, D. R. (2003). Evaluation of Control Measures Implemented in the Severe Acute Respiratory Syndrome Outbreak in Beijing, 2003. Journal of the American Medical Association. https://doi.org/10.1001/jama.290.24.3215.

Reynolds, D. L., Garay, J. R., Deamond, S. L., Moran, M. K., Gold, W., \& Styra, R. (2008). Understanding, compliance and psychological impact of the SARS quarantine experience. Epidemiology and Infection. https:// doi.org/10.1017/S0950268807009156.

Ribeiro, E., Sampaio, A., Gonçalves, M. M., Taveira, M. D. C., Cunha, J., Maia, Â., Matos, M., Gonçalves, S., Figueiredo, B., Freire, T., \& Soares, T. (2020). Telephone-based psychological crisis intervention: the Portuguese experience with COVID-19. Counselling Psychology Quarterly. https://doi.org/10.1080/095150 $\underline{70.2020 .1772200 .}$.

Salman, M., Asif, N., Mustafa, Z. U., Khan, T. M., Shehzadi, N., Hussain, K., Tahir, H., Raza, M. H., \& Khan, M. T. (2020). Psychological Impact of COVID-19 on Pakistani University Students and How They Are Coping. MedRxiv. https://doi.org/10.1101/2020.05.21.20108647.

Tang, B., Bragazzi, N. L., Li, Q., Tang, S., Xiao, Y., \& Wu, J. (2020). An updated estimation of the risk of transmission of the novel coronavirus (2019-nCov). Infectious Disease Modelling. https://doi.org/10.1016/j. idm.2020.02.001.

Thompson, G., McBride, R. B., Hosford, C. C., \& Halaas, G. (2016). Resilience Among Medical Students: The Role of Coping Style and Social Support. Teaching and Learning in Medicine. https://doi.org/10.1080 /10401334.2016.1146611. 
Ullah, R., \& Amin, S. (2020). The psychological impact of COVID-19 on medical students [Letter]. In Psychiatry Research. https://doi.org/10.1016/j.psychres.2020.113020.

Wang, C., Pan, R., Wan, X., Tan, Y., Xu, L., Ho, C. S., \& Ho, R. C. (2020). Immediate psychological responses and associated factors during the initial stage of the 2019 coronavirus disease (COVID-19) epidemic among the general population in China. International Journal of Environmental Research and Public Health. https:// doi.org/10.3390/ijerph17051729.

Wong, T. W., Gao, Y., \& Tam, W. W. S. (2007). Anxiety among university students during the SARS epidemic in Hong Kong. Stress and Health. https://doi.org/10.1002/smi.1116.

World Health Organization (WHO). (2020). No Title. https://twitter.com/WHO/ status $/ 1213523866703814656$ ? $=20$.

Xiao, C. (2020). A novel approach of consultation on 2019 novel coronavirus (COVID-19)-related psychological and mental problems: Structured letter therapy. In Psychiatry Investigation. https://doi. org/10.30773/pi.2020.0047. 